\title{
Agentes bacterianos en la sepsis neonatal. Cuidados Intensivos Neonatales Hospital Mario Catarino Rivas.
}

\author{
Bacterial agents in neonatal sepsis. Neonatal Intensive Care unit \\ Mario Catarino Rivas National Hospital
}

\section{Carlos Felipe Ponce, * Wilmer Alejandro Madrid,* Iván José Pineda. **}

\section{RESUMEN}

Antecedentes: Las infecciones nosocomiales son aquellas que se adquieren y se manifiestan luego de 48 horas de hospitalización Objetivo: Determinar los gérmenes aislados por cultivos de los recién nacidos diagnosticados como sepsis nosocomial en la unidad de cuidados intensivos neonatales (UCIN), Hospital Nacional Mario Catarino Rivas (HNMCR), en los meses de julio a septiembre del 2015. Pacientes y métodos: Estudio transversal, de los 443 pacientes ingresados a UCIN, 221 neonatos que desarrollaron infección posterior a 48 horas de internamiento. La información se obtuvo del expediente clínico y se procesó en el software estadístico Epi Info 3.02 Resultados: De los cultivos obtenidos; (165) 75\% resultaron positivos para algún germen específico. Los gérmenes aislados fueron; Pseudomona spp 71 (43\%) y Pseudomona aeruginosa 58 (35\%), haciendo un total de $78 \%$ de sepsis nosocomial por Pseudomona. Conclusión: La sepsis intrahospitalaria es un problema frecuente en UCIN, por lo tanto es necesario el cumplimiento de las normas de vigilancia y control de este tipo de infecciones.

\section{PALABRAS CLAVE}

Infección hospitalaria, medios de cultivo, Sepsis.

\section{ABSTRACT}

Background: Nosocomial infections are those that are acquired in a hospital and thy are

\footnotetext{
* Residente de segundo año del posgrado de Pediatría. Universidad Nacional Autónoma de Honduras, Valle de Sula, UNAH-VS.

** Pediatría Neonatólogo del Hospital Mario Catarino Rivas Dirigir correspondenciaa:cponce_xs@hotmail.com Recibido: 04 de octubre 2015 Aprobado: 04 de abril 2016
}

manifested 48 hours after hospitalization Objective: To determine the organism s isolated from cultures of newborns diagnosed as nosocomial sepsis in the neonatal intensive care unit (NICU) at the Mario Catarino Rivas National Hospital (HNMCR) between July and September 2015. Patients and methods: A transversal study was performed. 443 patients were admitted to NICU in the period of the stydy. The sample are 221 infants that developed sepsis after 48 hours of hospitalization, information was obtained from medical records, then it was processed in the statistical software Epi Info 3.02 Results: Of the cultures obtained $75 \%$ $(n=165)$ tested positive for a specific germ. Germs isolated were Pseudomonas spp 43\% $(n=71)$ and Pseudomonas aeruginosa 35\% $(n=58)$, making a total of $78 \%$ of nosocomial pseudomonas sepsis. Conclusion: Nosocomial sepsis continues to be a problem in the neonatal intensive care unit, therefore it is necessary to maintain continues monitoring and infection control.

\section{KEY WORDS}

Cross Infection, culture media, Sepsis.

\section{INTRODUCCIÓN}

En los últimos años debido a las mejoras y avances en la medicina, junto a amplios tratamientos de antibióticos, se extiende la estadía hospitalaria de los neonatos con bajo peso y prematuros. Estos son más susceptibles a infecciones nosocomiales. ${ }^{(1,2)}$ Las infecciones nosocomiales son aquellas que no están presentes o no se encuentran en incubación cuando se ingresó al paciente y que ocurren luego de 48 horas de haber sido ingresado. ${ }^{(3-5)}$ El término 
nosocomial viene del griego nosokomeian que significa nosos es enfermedad y komeian significa hospital. ${ }^{(4,5)}$

La incidencia de la sepsis neonatal varía de 7.1 a 38 por cada 1000 nacidos vivos en Asia, de 6.5 a 23 por 1.000 nacidos vivos en África, y de 3,5 a 8,9 por cada 1000 nacidos vivos en América del Sur y el Caribe. En comparación, las tasas reportadas en Estados Unidos y Australasia varian desde 1,5 a 6 por 1000 nacidos vivos. ${ }^{(5)}$

El Instituto Nacional de Salud y de Desarrollo Humano "Neonatal Network" indica que el 29\% de los neonatos entre $25-28$ semanas y el $46 \%$ menor de 25 semanas adquieren algún tipo de infección nosocomial, pudiendo causar fallecimiento hasta en un $50 \%$ de quienes las adquieren. ${ }^{(6)}$

Según la Sociedad de Enfermedades Infecciosas Pediátricas (JPIDS, por sus siglas en inglés) la incidencia afecta alrededor de $2.43 \%$ de los recién nacidos ingresados en las UCIN. El gérmen aislado con frecuencia es el estafilococo coagulasa negativos y de ellos el S. Epidermidis el más frecuente. ${ }^{(7)}$ Usualmente las bacterias gram positivos predominan sobre los gram negativos. Dependiendo del tiempo de estancia hospitalaria el germen etiológico aislado varía, por ejemplo, en los cincuenta el más frecuente era el estafilococo aureus, en los sesenta eran las Pseudomonas y Escherichia coli y a partir de los setenta se ha mantenido el estafilococo coagulasa negativo en un 20-30\%. Otros gérmenes como ser candida spp, particularmente la Albicans y Parapsilosis causan el $10 \%$ de las infecciones nosocomiales. ${ }^{(8-10)}$

El objetivo del estudio fue determinar los gérmenes más frecuentes aislados en los cultivos de los recién nacidos diagnosticados como sepsis nosocomial en la UCIN, Hospital Mario Catarino Rivas, en los meses de julio a septiembre 2015.

\section{MATERIALES Y MÉTODOS}

Se realizó un estudio descriptivo retrospectivo transversal. El universo fueron todos los recién nacidos ingresados a la sala de UCIN del HMCR durante julio a septiembre del 2015, correspondiendo a una población de 443 neonatos en un período comprendido entre julio-septiembre 2015, la muestra es de 221 que cumplían con los criterios de inclusión: diagnóstico de sepsis 48 horas después de su hospitalización en la UCIN, sin distinción de patología concomitante y que se haya registrado la toma de algún cultivo; y los criterios de exclusión: Recién nacidos con diagnostico diferente a sepsis, pacientes sin cultivos tomados o que aún no se haya obtenido reporte de laboratorio en las fechas establecidas del estudio.

Se consideró como sepsis nosocomial a la presencia de al menos un agente aislado por distintos métodos de cultivo (hemocultivo, urocultivo, cultivo de líquido cefalorraquídeo, cultivo de punta de catéter), que por definición son infecciones que no están presentes o no se encuentran en incubación cuando se ingresó el paciente y que ocurren luego de 48 horas de haber sido ingresado de acuerdo con el Centro de Prevención y Control de Enfermedades de Estados Unidos, para tener una mejor identificación de los casos. ${ }^{(5)}$

Los datos obtenidos de los expedientes clínicos se consignaron en un cuestionario de recolección que incluía nombre, sexo, edad, tipo de cultivo, microorganismo causal, sensibilidad y resistencia del microorganismo, posteriormente se transcribió en una base de datos en Epilnfo 3.02 con el posterior análisis estadístico, se aplicaron medidas de tendencia central para la presentación de los datos se utilizó la hoja de Microsoft Excel 2013.

\section{RESULTADOS}

Se encontró que de los 221 neonatos, en 165 (75\%) los cultivos obtenidos resultaron positivos para algún germen específico.

(Ver tabla No. 1) 
Tabla No. 1: Resultados de cultivos tomados

\begin{tabular}{ccc}
\hline Cultivos & $\mathbf{n}$ & Porcentaje \\
\hline Positivos & 165 & $75 \%$ \\
Negativos & 56 & $25 \%$ \\
Total & 221 & $100 \%$ \\
\hline
\end{tabular}

Fuente: Investigación, infecciones nosocomiales. UCIN-HMCR, 2015

Los cultivos realizador fueron; hemocultivo (176) $79 \%$ de los casos, punta de catéter 18 (8\%). (Ver Tabla No. 2)

Tabla No. 2: Tipo de cultivos Tomados

\begin{tabular}{ccc}
\hline Tipo de Cultivo & $\mathbf{n}$ & Porcentaje \\
\hline Hemocultivo & 176 & $79 \%$ \\
Punta de catéter & 18 & $8 \%$ \\
Urocultivo & 14 & $6 \%$ \\
Otros* & 9 & $5 \%$ \\
LCR & 4 & $2 \%$ \\
Total & 221 & $100 \%$ \\
\hline
\end{tabular}

* Otros: Secreción Nasal, secreción ocular, Secreción de Herida quirúrgica, secreción de absceso.

Fuente: Investigación, infecciones nosocomiales. UCIN-HMCR, 2015

Los gérmenes aislados fueron; Pseudomonas spp 71 (43\%) y Pseudomonas aeruginosas 58 (35\%), haciendo un total de $78 \%$ de sepsis nosocomial por Pseudomonas. (Ver Tabla No. 3)

Tabla No. 3: Tipo de Germen aislado

\begin{tabular}{lcc}
\hline Germen aislado & $\mathrm{n}$ & Porcentaje \\
\hline Pseudomonas spp & 71 & $43 \%$ \\
Pseudomonas aeruginosa & 58 & $35 \%$ \\
Staphylococcus spp & 14 & $8 \%$ \\
Klebsiella pneumoniae & 9 & $6 \%$ \\
Bacilo Gram negativo No & & \\
$\quad$ fermentador & 7 & $4 \%$ \\
Staphylococcus aureus & 5 & $3 \%$ \\
Corynebacterium spp & 1 & $1 \%$ \\
$\quad$ Total & 165 & $100 \%$ \\
\hline Fuente: Investigación, infecciones nosocomiales. UCIN-HMCR, \\
$2015 \quad$
\end{tabular}

La mayor sensibilidad reportada para el germen aislado fue a la familia de las quinolonas como ser Levofloxacina y ciprofloxacina con $34 \%$ y $23 \%$ respectivamente (haciendo un total de $57 \%$, de gérmenes sensibles a quinolonas) (Ver Gráfica No. 1)

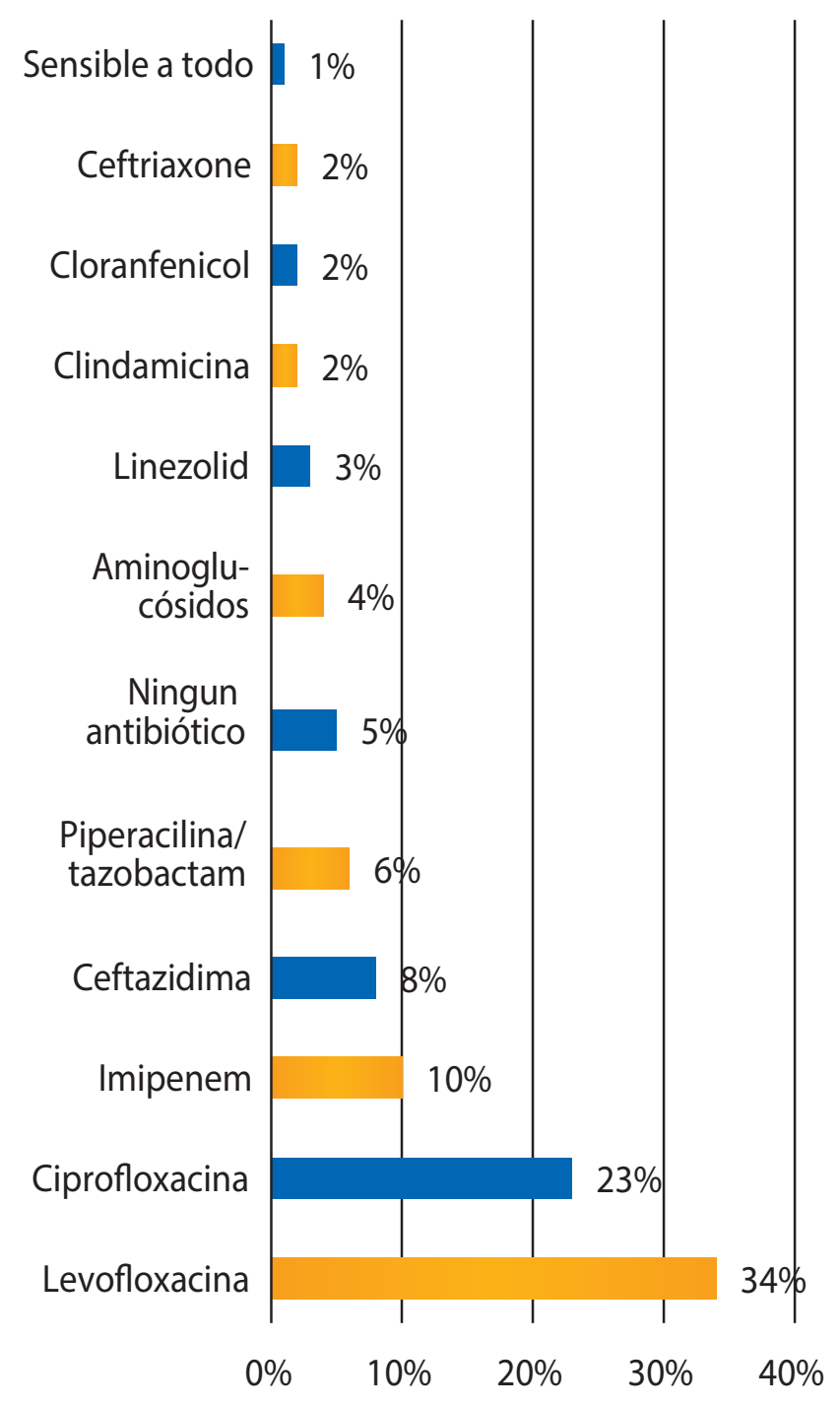

\section{Gráfica No. 1: Sensibilidad antibiótica según gérmenes aislados.}

Fuente: Investigación, infecciones nosocomiales. UCIN-HMCR, 2015.

La resistencia de los microorganismos según antibiogramas reportados se trataba de gérmenes carbapenemasas en un $30 \%$ y Betalactamasa tipo AmpC en un 41\%. (Ver Gráfica No. 2) 


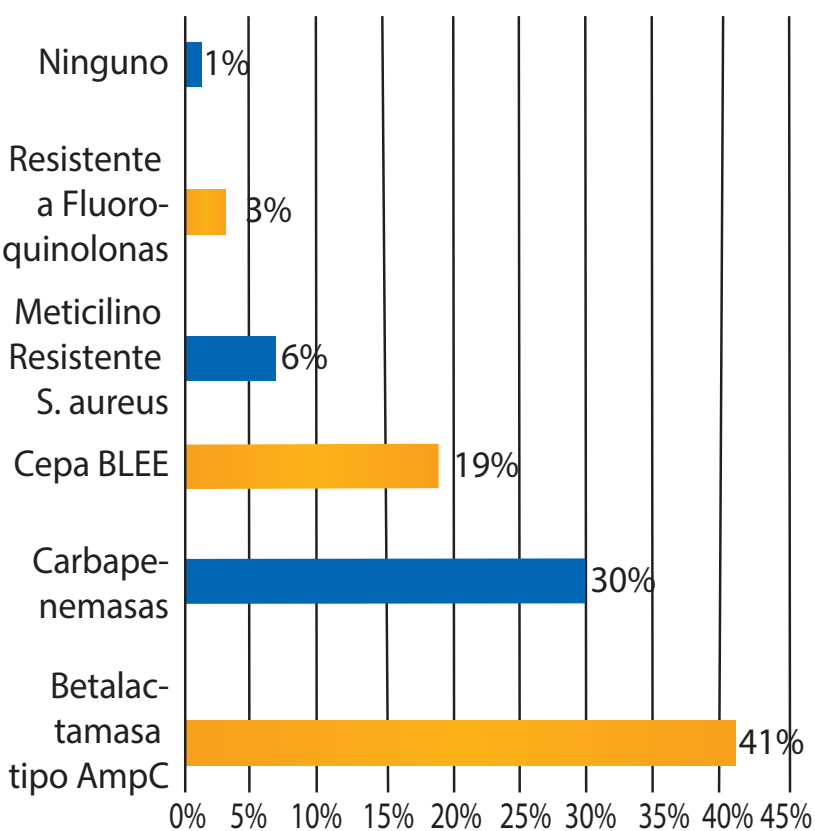

\section{Gráfica No. 2: Resistencia antibiótica según gérmenes aislados.}

Fuente: Investigación, infecciones nosocomiales. UCIN-HMCR, 2015.

\section{DISCUSIÓN}

Uno de los principales problemas clínicos en la sepsis neonatal, es su detección temprana. ${ }^{(10)}$ En la UCIN del HNMCR se tiene alta frecuencia de recién nacidos (RN) con datos clínicos sugestivos de sepsis, con signos de respuesta inflamatoria sistémica, sin confirmación microbiológica por aislamiento.

Los cultivos positivos obtenidos en este estudio fueron del $75 \%$ para algún germen especifico, una cifra alarmante si comparamos datos estadísticos de países desarrollados donde refiere que a pesar del subregistro de infecciones, se ha encontrado que $6.2 \%-33 \%$ de los neonatos admitidos se les ha diagnosticado alguna infección durante su estadía; (11) datos similares fueron descritos en 8 unidades de cuidados intensivos neonatales en Hospitales de Colombia donde la incidencia de infecciones nosocomiales por admisión es variable de 6.2 a $33 \%{ }^{(12,13)}$ en los EE.UU., la incidencia de infección nosocomial en UCIN es menor encon- trándose entre 2 y $24.6 \% .^{(14,15)}$

El gold estándar para diagnóstico de sepsis nosocomial continúa siendo la presencia de por lo menos un cultivo positivo. ${ }^{(16,17)}$ En este estudio se documentó, que el mayor porcentaje de infecciones fue confirmado por hemocultivo, coincidiendo con la literatura internacional mencionada.

El valor de identificar la prevalencia de cepas resistentes es imprescindible para el manejo empírico y definitivo de pacientes con infecciones intrahospitalarias, ${ }^{(17)}$ un hallazgo claro en esta investigación fue la demostración de la bacteria Gram-negativa, aeróbica, Pseudomonas incluyendo las dos cepas aeruginosa y spp representando el $78 \%$ de las muestras, siendo considerado como uno de los mayores gérmenes oportunistas. Esto se traduce en un aumento de morbilidad, mortalidad y gasto por hospitalización. Contrastando con lo reportado por la Sociedad de Enfermedades Infecciosas Pediátricas, que menciona que solamente el $20 \%$ a $30 \%$ de los casos de infección nosocomial son causado por gérmenes gram-negativos; ${ }^{(17)}$ colocando a los cocos gram-positivos como responsables en mayor proporción de infecciones nosocomiales, incluyendo Staphylococcus aureus meticilino resistente (SARM), Staphylococcus coagulasa negativos (SCN) predominantemente epidermidis, y enterococos resistentes a vancomicina. ${ }^{(17,18)}$

Los mecanismos intrínsecos de la bacteria predominante en este estudio explican gran parte su sensibilidad o resistencia cruzada a diferentes clases de antibióticos; se encontró que de los 165 cultivos positivos, 57\% eran sensibles a quinolonas y únicamente $6 \%$ sensible a piperacilina más tazobactam; estos hallazgos en antibiograma difieren de otros estudios como los publicados en Gran Bretaña por Sánchez-Carrillo et al y Naze et al., donde mencionan que la Piperacilina/Tazobactam mantiene susceptibilidad frente a Pseudomona. ${ }^{(17)}$ Además debemos considerar que el uso 
de quinolonas en neonatos según literatura publicada en Archives of Disease in Childhood han mostrado diversos mecanismos moleculares como responsable de eventos adversos; tales como la inhibición de la síntesis de colágeno y glucosaminoglicanos, inhibición de la función mitocondrial, generación de radicales libres, estrés oxidativo y quelación de iones de magnesio que culminan en daño al cartílago y tendones en los neonatos, sin embargo en el HNMCR se utilizan debido a poca disponibilidad de otros antibióticos, también no hay estudios de seguimiento en estos pacientes que pudieran demostrar efectos adversos a largo plazo. ${ }^{(18,19)}$

El uso indiscriminado de los antibióticos y la presión selectiva ambiental, realizada por antisépticos y desinfectantes, ha generado una respuesta de supervivencia en los microorganismos, que los capacita para evadir con eficiencia la acción bactericida de algunos agentes. ${ }^{(20)}$ En el $41 \%$ de los cultivos reportados se observó que la resistencia de los microorganismos estuvo dada por gérmenes Betalactamasa tipo AmpC, estos hallazgos no concuerdan con los encontrados por Foca $\mathrm{M}$ y Jacob $K$ en su estudio Infección Endémica por Pseudomonas en una UCIN en Hospital pediátrico de Nueva York, donde solo el $18 \%$ de los microorganismos presentaron similar resistencia, por lo mencionado anteriormente estos patógenos son de alta prioridad, por representar problemas clínicos o de salud pública relevante, además de ser muy limitadas las alternativas terapéuticas en estos casos. ${ }^{(21)}$

En conclusión, la sepsis intrahospitalaria sigue siendo un problema en la UCIN, por lo tanto, es necesario el cumplimiento de las normas de vigilancia y control de este tipo de infecciones. La mayoría de los pacientes presenta patologías de cierto grado de complejidad que ameritan múltiples procesos invasivos, tanto para el diagnóstico como para el manejo exponiéndolos a múltiples factores de riesgo, lo que aumenta la probabilidad de adquirir infecciones intrahospitalarias.

Se recomienda que para el control de las infecciones nosocomiales participe un comité multidisciplinario que mantenga políticas y una vigilancia activa del programa, la prevención debe ser una prioridad y se puede lograr si se tiene un adecuado conocimiento de la epidemiología, tratamiento y del manejo de los protocolos en cada institución, además la estricta vigilancia debe incluir practica adecuada del lavado de manos, promoción de la utilización de la leche materna, garantizando la obtención y almacenamiento sanitario de la misma, proteger la piel del recién nacido para promover su maduración evitando en lo posible pruebas de laboratorio que requieran venopunción, reducir al mínimo los días de intubación y reservar el uso de líneas centrales solo cuando la terapia intravenosa sea de larga duración.

\section{BIBLIOGRAFÍA}

1. Kamath S, Mallaya S, Shenoy S. Nosocomial infectios in Neonatal Intensive Care Units: Profile, Risk factor, Assessment and Antibiogram. Indian Journal of Pediatrics. [Revista en internet] 2010 [Citado 28 Julio 2015]; 77(1): 37-39. doi: 10.1007/s12098 -010-0005-5. Disponible en: http://www. ncbi.nlm.nih.gov/pubmed/20135266.

2. Polin RA, Saiman L. Nosocomial Infections in the Neonatal Intensive Care Unit. Neoreviews. [Revista en internet] 2003 [Citado 2 Agosto 2015]; 4(3):81-89. Disponible en: http://www.ohsu.edu/xd/health/services/ doernbecher/research-education/educa tion/residency/upload/res_lounge_noso comial-infections.pdf.

3. Polin RA, Committee of fetus and newborn. Management of Neonates with suspected 
or proven early-onset bacterial sepsis. Pediatrics. [Revista en internet] 2012 [Citado 30 Julio 2015]; 129(5): 1006-1015. doi: 10.1542/peds.2012-0541. Disponible en: http://www.ncbi.nlm.nih.gov/pubmed/ 22547779.

4. Bhatti M, Chu A, Hageman JR, Schreiber M, Alexander $\mathrm{K}$. Future directions in the evaluation and management of neonatal sepsis. Neoreviews. [Revista en internet] 2012 [Citado 5 Agosto 2015]; 13(2): 103110. DOI: 10.1542/neo.13-2-e103.

Disponible en: http://pediatrics.uchicgo. edu/Research/Publications/Bhatti.Chu\%20 Future\%20Directions\%20NeoReviews $\% 2020$ 12.pdf.

5. S Vergnano, M Sharland, P Kazembe, C Mwansambo, PT Heath. Neonatal Sepsis: an international perspective. Arch Dis Child Fetal Neonatal 2005; 90:220- 224. doi: 10.11 36/adc.2002.022863.

6. Eraso Jenny, Troncoso Gloria, Álvarez Marta, Ibáñez Milcíades. Comportamiento epidemiológico de la sepsis nosocomial en la Unidad de Recién Nacidos de la Fundación Cardioinfantil. Rev. Cienc. Salud [serial on the Internet]. $2008 \mathrm{Apr}$ [citado 30 de marzo 2016 ]; 6( 1): 36-49. Disponible en: http:// www.scielo.org.co/scielo.php?script $=$ s ci_arttext\&pid=S1692-72732008000100 005\&lng=en.

7. Borghesi A, Stronati M. Strategies for the prevention of hospital-acquired infections in the neonatal intensive care unit. Hospital Infection Society. [Revista en internet] 2009 [Citado 18 Agosto 2015]; 71(1):96-97. doi: 10.1016/j.jhin.2008.09.019. Disponible en: http://www.ncbi.nlm.nih.gov/pubmed/ 19013668.

8. Venakatesh M, Flores A, Luna RA, Versalovic J. Molecular microbiological methods in the diagnosis of neonatal sepsis. Expert review of anti-infective therapy. [Revista en internet] 2010 [Citado 13 Agosto 2015]; 8(9): 1037-1048. doi: 10.1586/eri.10.89.

Disponible en: http://www.ncbi.nlm.nih. gov/pubmed/20818947

9. Foca M, Jacob K, Whittier S, Della P, Factor S, Rubenstein D, et al. Endemic Pseudomonas aeruginosa infection in a neonatal intensive care unit. New England Journal of Medicine. [Revista en internet] 2000 [Citado 2 Septiembre 2015]; 343(1): Pp. 695-700. DOI: 10.1056/NEJM200009073431004. Disponible en: http://www.nejm.org/doi/full/10.10 56/NEJM200009073431004\#t=articleTop.

10. Mendoza L, Osorio M, Fernández M, Henao C, Arias M. Tiempo de crecimiento bacteriano en hemocultivos en neonatos. Revista Chilena de Pediatría. [Revista en internet] 2015 [Citado 10 Octubre 2015]; 86(5): 337-344. doi:10.1016/j.rchipe.2015.07.004. Disponible en: http://www.sciencedirect. com/science/article/pii/S0370410615 001400.

11. Lodha R, Natchu UC, Nanda M, Kabra SK. Nosocomial infections in pediatric intensive care units. Indian Journal of Pediatrics. [Revista en internet] 2001 [Citado 5 Septiembre 2015]; 68(11):1063-1070. DOI: 10. 1007/BF02722358. Disponible en: nosoco mial-infections-in-pediatric-intensive-ca re-units-6sxGqLrPtF.

12. Garner JS. Guideline for isolation precautions in hospitals. The Hospital infection control practices Advisory committee. Infect Control Hosp Epidemiol. [Revista en internet] 1996 [Citado 10 Septiembre 2015]; 17(1): Pp. 53-80. Disponible en: http://wonder.cdc.gov/wonder/prevguid/ p0000419/p0000419.asp.

13. Taeusch HW, Ballard RA, Gleason CA. Avery $s$ diseases of the newborn. Eighth edition. Philadelphia: Elsevier; 2005. 
14. Hernández HG, Castañeda JL, González N. Infecciones nosocomiales asociadas a métodos invasivos en un hospital pediátrico de alta especialidad. Revista de Enfermedades Infecciosas en Pediatría. [Revista en internet] 2011 [Citado 26 Septiembre 2015] 22(88). Disponible en: www. files.sld.cu/ anestesiologia/files/2011/11/infecciones -nosocomiales.

15. Vergnano S, Sharland M, Kazembe P, Mwansambo PT. Heath. Neonatal Sepsis: an international perspective. Archives of Disease in Childhood Fetal and Neonatal Edition. [Revista en internet] 2005 [Citado 13 Septiembre 2015]; 90(3):220-224. Disponible en: http://www.ncbi.nlm.nih.gov/pubmed/ 15846011.

16. Guías clínicas del departamento de neonatología 2011. Hospital Infantil de México "Federico Gómez".

17. Cercenado E, Vicente MF, Díaz MD, Sánchez-Carrillo C, Sánchez-Rubiales M. Characterization of clinical isolates of beta -lactamase negative highly-ampicillin-re sistant Enterococcus faecalis. Antimicrob Agents Chemother. 1996; 34. 40:2420-2.

18. Edmond K, Zaidi A. New Approaches to Preventing, Diagnosing and Treating Neo- natal Sepsis. PLoS Med. [Revista en internet] 2010 [Citada 10 Septiembre 2015]; 7(3):1000213. doi: 10.1371/journal.pmed. 1000213. Disponible en: http://www.ncbi. nlm.nih.gov/pmc/articles/PMC2834705/.

19. Camacho-Gonzalez A, Spearman P, Stoll B. Neonatal Infectious Diseases: Evaluation of Neonatal Sepsis. Pediatr Clin North Am. [Revista en internet] 2013 [Citado 29 Septiembre 2015]; 60(2): 367-389. DOI: 10.1016/j.pcl.2012.12.003. Disponible en: http://www.researchgate.net/publication/ 236037485_Neonatal_Infectious_Disea ses_Evaluation_of_Neonatal_Sepsis.

20. Marie Agnes Simonin. Antimicrobial Agents and Chemotherapy, Proteoglycan and Collagen Biochemical Variations during Fluoroquinolone-Induced Chondrotoxicity in Mice, Antimicrob Agents Chemother. 1999 Dec; 43(12): 2915-2921.

21. Tripathi N, Cotten, CM, Smith PB. Antibiotic Use and Misuse in the Neonatal Intensive Care Unit. Clin Perinatol. [Revista en internet] 2012 [Citado 2 Octubre 2015]; 39(1):61-68. DOI: http://dx.doi.org/10.10 16/j.clp.2011.12.003. Disponible en: http:// www.perinatology.theclinics.com/arti cle/S0095-5108(11)00130-8/pdf. 